Review

\title{
Mesenchymal Stem Cell Derived Exosomes in Cancer Progression, Metastasis and Drug Delivery: A Comprehensive Review
}

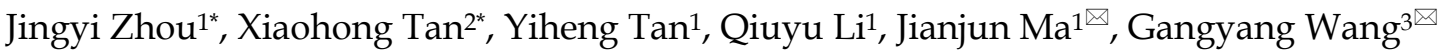 \\ 1. Human Anatomy Laboratory, School of Basic Medicine, Xinxiang Medical University, Henan, 453003, China \\ 2. The Third Affiliated Hospital of Xinxiang Medical University, Henan,453003, China \\ 3. Department of Orthopaedics, Shanghai Bone Tumor Institute, Shanghai General Hospital, Shanghai Jiao Tong University School of Medicine, Shanghai, \\ 200080, China \\ *These authors have contributed equally to this work
}

$\triangle$ Corresponding authors: Jianjun Ma, MD, Human Anatomy Laboratory, School of Basic Medicine, Xinxiang Medical University, 01 Jinsui Avenue, Xinxiang 453000, China. Email: mij19631030@163.com. Gangyang Wang, MD, Ph.D., Department of Orthopaedics, Shanghai Bone Tumor Institute, Shanghai General Hospital, Shanghai Jiao Tong University School of Medicine, Shanghai 20000, China. Email: wgy74521@sjtu.edu.cn or gangyang_wang@163.com

(C) Ivyspring International Publisher. This is an open access article distributed under the terms of the Creative Commons Attribution (CC BY-NC) license (https://creativecommons.org/licenses/by-nc/4.0/). See http://ivyspring.com/terms for full terms and conditions.

Received: 2018.02.05; Accepted: 2018.07.14; Published: 2018.08.06

\begin{abstract}
With the development of cancer treatments, it has become a popular research focus that mesenchymal stem (or stromal) cells (MSCs) have the functional mechanisms that influence cancer progression. One of the underestimated mechanisms is secretion of highly specialized double-membrane structures called exosomes. Mesenchymal stem cells generate several exosomes that may act as paracrine mediators by exchanging genetic information. MSC-derived exosomes are microvesicles ranging from approximately 60-200 nm in size and detected in various body fluids. It has been demonstrated that MSC-derived exosomes are involved in tumor growth, angiogenesis, metastasis, and invasion. Furthermore, emerging evidence suggests that as natural nanocarriers, MSC-exosomes are responsible for multidrug resistance mechanisms, reverse effect of radiation injury, and immune regulation, which can be used in clinical applications for cancer therapy. The present review aims to briefly describe the properties and biological functions of MSC-exosomes in cancer progression and its possible clinical applications in the future.
\end{abstract}

Key words: Mesenchymal stem cell, exosomes, cancer therapy

\section{Introduction}

Mesenchymal stem (or stromal) cells (MSCs) have emerged as a potential solution for tissue repair and wound healing [1]. Recent data imply that MSCs mediate their therapeutic functions in a paracrine rather than a cellular manner [2]. At present, the only human cell type known to have a scalable capacity to mass produce exosomes is MSC and MSC is the ideal cell candidate for the mass production of exosomes for drug delivery [3]. MSCs are multipotent fibroblast-like cells that reside in many adult tissues such as adipose tissue [4], periosteum liver, lung, spleen, muscle connective tissue, amniotic fluid, placenta, and aborted fetal tissues [5-11]. In vitro, they are representative expanded as plastic adherent cells [2]. Because of their low immunogenicity, MSCs can suppress the function of various immune effector cell types and promote immune regulatory functions [12, 13]. According to these features, MSCs became a desirable cell source in regenerative medicine and immune therapy [2].

Exosomes, like the intraluminal vesicles, range approximately from $30-100 \mathrm{~nm}$ in diameter secreted by live cells and were first observed in the early 1980s[14]. Exosomes have been found in numerous body fluids, including blood, amniotic fluid, urine, malignant ascites, cerebrospinal fluid, breast milk, 
saliva, lymph, and bile, under both healthy and morbid conditions [15-17]. Exosomes have an extracellular membrane vesicle structure composed of a phospholipid bilayer membrane [18]. Recent information from different cell type reveals that exosomes contain4, 563 proteins, 194 lipids, 764 microRNA and 1639 mRNA [19, 20]. Also, a multitude of pathways can be activated by exosomes because of cellular interactions with exosomal molecules, including mRNAs, miRNAs, and proteins (e.g., heat shock proteins [HSPs] and adhesion molecules) [21].

Generally, exosome biogenesis is composed of two steps, the inward budding of membranous vesicles of endosomes and their release into a structure known as a multivesicular body (MVB), while exosomes are mainly secreted by two different mechanisms, constitutive release via the Trans-Golgi network and inducible release [14]. Exosomes interact with target cells, including receptors, endocytosis, fusion with plasma membrane or the release of their cargo [22]. An additional figure file shows this in more detail (Figure 1). In this way, exosomes function as natural nanocarriers, allowing the transport of the bioactive factors they carry to a recipient cell [23]. Remarkably, MSC-derived exosomes can reach to most tumor territories and provide a suitable microenvironment for cancer development, such as cell proliferation, drug resistance, angiogenesis and metastasis, immune modulation. Here we will summarize recent studies on the role of MSC-derived exosomes play in cancer development, the mechanism that MSC-exosomes transport cancer drug resistance, and discuss their application to diagnostics and therapy.

\section{Characterization of Mesenchymal Stem Cell-Derived Exosomes}

At present, MSCs are known as the only human cell type to have a scalable capacity to mass produce exosomes [24]. MSC-derived exosomes were first investigated in 2010 in a mouse model of myocardial ischemia/reperfusion injury [25]. The characterization of exosomes has also been found to correlate with its cell origin. For example, all of the MSC-derived exosomes expressed markers CD63 CD9 and CD81 $[26,27]$, but the human umbilical cord MSC-EXOs especially expressed exosomal markers, such as Hsp70 and TSG101 proteins, and also some adhesion molecules, such as CD29, CD44 and CD73 are expressed on the membrane of MSCs [28-30]. Hence, identifying the source of exosomes and isolating them from extracellular matrix through their unique features could be a way to approach. Under transmission electron microscope, the MSC-derived exosomes still exhibited the characteristic round morphology with heterogeneous size. And the average size of MSC-exosomes was $48.72 \pm 2.7 \mathrm{~nm}$ [31], the major peak in particle size of MSC-exosomes was at $65-75 \mathrm{~nm}$ and the overall size distribution ranged between 60 and $200 \mathrm{~nm}$ [26]. In addition, Nakamura $Y$ et al. examined miRNA in MSC-exosomes using a

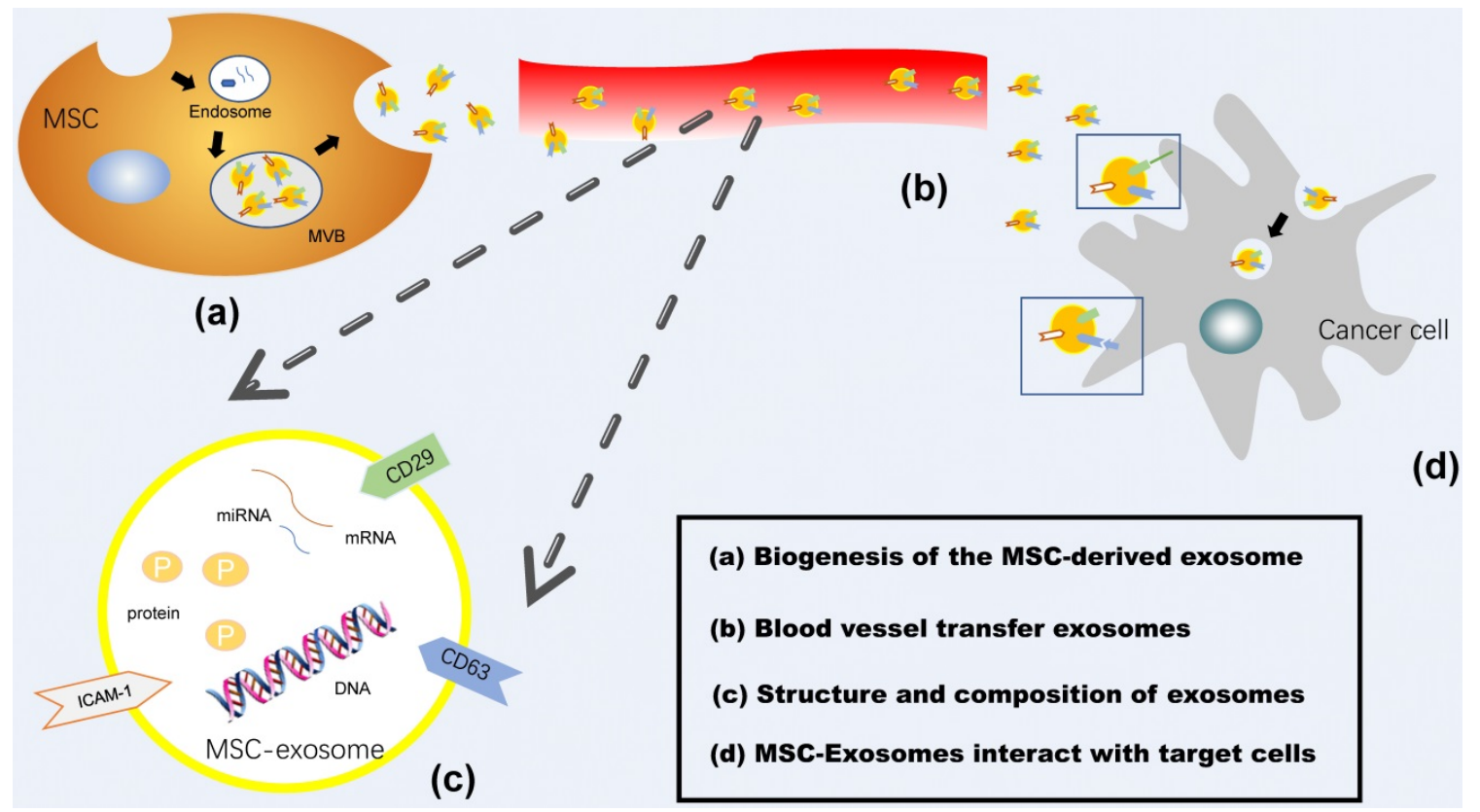

Figure 1. Mesenchymal Stem Cell-derived Exosomes. (a) Biogenesis of the MSC-derived exosome: Exosomes are generated by an endocytic process as follows: (1) inward budding membranous vesicles with an incorporation of protein wrapped into endosome; (2) their release into a structure known as a multivesicular body (MVB); ( 3 ) fusion of MVB with mesenchymal stem cell membrane and release of exosomes into the extracellular space. (b) MSC-Exosomes are transferred via the blood vessel to the target cells. (c) Structure and composition of exosomes: Exosomes are phospholipid bilayer membrane containing proteins and genetic materials such as DNA, mRNA, and miRNA. (d) MSC-Exosomes interact with target cells, including receptors, endocytosis, fusion with plasma membrane. 
NanoString miRNA analysis, which listed the top 20 miRNAs according to their expression levels in MSC-exosomes and miR-21, an antiapoptotic miRNA [32], was detected at the highest concentrations in MSC-exosomes [26]. Furthermore, Lai $R$ et al. have found that the predominant feature of MSC-exosome proteome was the presence of all seven $\alpha$ and seven $\beta$ chains of the $20 \mathrm{~S}$ proteasome, and 379,432 , and 420 unique proteins have been detected by liquid chromatography-mass spectrometry/mass spectrometry [33]. All these studies show how special the exosomes are; their unique features make them a vital component of cancer procession, it is possible that changing the phenotype of MSC-exosomes then they can combine with designated receptor cells and exert specific effects.

\section{MSC-exosomes in cancer procession}

The number of detected exosomes in patients diagnosed with cancer was found to be increased compared to healthy controls. This finding indicated the significant role of exosomes in the development and progression of various types of cancer [34]. Growing evidence suggests that MSC-exosomes could transfer proteins, messenger RNA, and microRNA to recipient cells then exert various effects on the growth, metastasis, and drug response of different tumor cells [35]. And previous studies have demonstrated that mesenchymal stem cells generate several exosomes that may act as paracrine mediators by exchanging genetic information [36, 37]. Therefore, understanding the underlying and complex MSC-exosome mediating mechanisms between the tumor cell and their microenvironment in cancer progression is critical to discover the novel therapeutic approach to cancer.

\section{Tumor growth}

MSC-derived exosomes, as paracrine factors, transfer their contents to neighboring tumor cells or induce the phenotypic modifications in recipient cells [38], which could influence tumor progression in vitro and in vivo. To understand the mechanism responsible for the effects of MSC-exosomes on tumor growth in vivo, $Z h u$ et al, subcutaneously co-implanted human gastric and colon cancer cell lines with MSCs or MSC-exosomes into BALB/c-nu/nu mice, then an increased proliferative capacity was observed in the MSC-exosomes co-implantation group with tumor cells [39], their results show an increasing expression of $\mathrm{Bcl}-2$, phosphorylated ERK1/2, CXCR4 and VEGF proteins and a -SMA, CXCR4, VEGF and MDM2 mRNA, which are known to be vital to tumor growth and angiogenesis. Moreover, the study shows that
MSC-exosomes strongly activate VEGF and CXCR4 expression by activating ERK1/2 and p38 MAPK pathways, it indicated that MSC-exosomes did not promote tumor growth directly but enhanced a pro-angiogenic program, induced a richer blood supply, and then strengthen the capacity for tumor proliferation [39]. Furthermore, Qi et al. found that human bone marrow MSC-derived exosomes activated the Hedgehog signaling pathway in recipient osteosarcoma and gastric cancer cells line and induced tumor progression [40]. However, in multiple myeloma (MM) cell, MM BM-MSC-derived exosomes are absorbed by MM cells, which have higher levels of oncogenic proteins, cytokines, and adhesion molecules compared with normal BM-MSC exosomes, these contents lead to modulation of tumor growth in vivo; therefore MM BM-MSC-derived exosomes promoted MM tumor growth, while normal BM-MSC exosomes inhibited the growth of MM cells [41]. Yang et al. found that MSC-derived exosomes contained matrix metalloproteinase-2 or MMP-2 enzyme could alter cellular functionalities and provide the capability to re-organize the tumor microenvironment [42], and that is a novel approach to improve tumor growth. MSC-exosomes also act as carriers that transport tumor supportive proteins, miRNA, lipids, and metabolites, which plays an essential role in supporting breast cancer growth [43]. On the contrary, MSC-exosomes can also significantly down-regulated the expression of vascular endothelial growth factor (VEGF) in breast cancer cells, in vitro and in vivo, which is responsible for the anti-angiogenic effect of MSC-derived exosomes, and suppress the tumor growth in breast cancer [44]. Indeed, exosomes from human BM-MSCs transfer exosomal miR-100 and modulate the mTOR/HIF-1a signaling axis, to down-regulate VEGF expression in breast cancer-derived cells, which would affect the vascular behavior of endothelial cells and suppress the growth of breast cancer in vitro [45]. Another study indicated that MSCs packaged miR-146b into secreted exosomes, then MSC exosomes carrying miR-146b delivered the miRNA into glioma cells, which means MSC-exosomes could be used as a vehicle to transfer anti-tumor miRNAs (miR-146b) [46], and reduce glioma xenograft growth in a rat model of primary brain tumor [47].

Taken together, MSC-exosomes can affect tumor growth in both support and inhibition ways, which depends on the paracrine functions of MSC. It is possible that variable timing of MSC growth, composition of culture media, and passages of MSC used lead to different exosomes then represent the conflicting data $[48,49]$.Therefore, it is necessary to control growth condition of MSC and make sure they 
could obtain consistent results with their exosomes.

\section{Tumor angiogenesis}

There have been studies on the role of MSC-exosomes in angiogenesis, the cancer cells derived from the exosomes contain interleukin-6 (IL-6) and potent pro-angiogenic factors, vascular endothelial growth factor (VEGF), other molecules able to enhance organization and endothelial cell in tubule-like structures[50, 51]. Many studies have shown that MSCs plays an important role in angiogenesis, but the role of MSC-exosomes in angiogenesis is still controversial, and other studies have suggested that the external secretion of the body has been produced by blood vessels. To analyze the effects of MSC-exosomes on angiogenic activity in vitro, it significant stimulation of angiogenesis to prevent tumor necrosis. VEGF (Vascular endothelial growth factor) often regulated by MMPs (matrix metalloproteases), HIF-1a (hypoxia-inducible factor), and a wide range of other metabolic regulators and transcription factors $[1,52]$, such as ROS. Continuous ROS (reactive oxygen species) production promotes pathological angiogenesis operating mainly on the VEGF signaling pathway [53]. In the tumor, tumor mass and stromal cells produce substantial amounts of ROS, and the endogenous ROS production by the tumor cells regulates angiogenesis [54].

There are also data showing that MSC-exosomes secretion inhibits the formation of VEGF. Although the high concentration of MSC-exosomes effectively suppressed tumor growth and angiogenesis, in the beginning, anti-tumor effects of MSC-exosomes were not weakened over time. Thus, MSC-derived exosomes can be an effective anti-angiogenetic agent for anti-tumor therapy.

\section{Tumor metastasis and invasion}

Tumor metastasis and invasion require formation of a favorable niche, which is a specific microenvironment that promotes tumor cell viability, proliferation, metastasis and invasion [55]. Several studies have examined the role of MSC-derived exosomes in metastasis, invasion and the formation of a pre-metastatic niche. In breast cancer cell line MCF7, after treatment with MSC-exosomes, MCF7 breast cancer cells exhibited an enhanced migratory capacity. Moreover, MSC-exosome treatment led to a significant increase in $\beta$-catenin mRNA and protein levels. The expression of WNT target genes such as Axin2 and Dkk1 was also increased. The results demonstrated that MSC-exosomes promote MCF7 migration through the activation of the Wnt signaling pathway [56]. And this is the first report about MSC-exosomes promote tumor migration. A year after that, Wang et al. discovered that miRNAs were packaged into exosomes secreted by gastric cancer-MSC, delivered into gastric cancer cells and promoted gastric cancer metastasis [57]. After delving, they found the expression of miR-221 was significantly higher than other miRNA contents existed in GC-MSC-exosomes, and it's known that high expression of miR-221 showed a significant correlation with advanced tumor-node-metastasis stage, local invasion and lymphatic metastasis [58]. Besides, MSC-exosome increased the expression of mesenchymal markers and reduced the expression of epithelial markers in gastric cancer cells and then induce the epithelial-mesenchymal transition, which enhanced the migration and invasion of gastric cancer HGC-27 cells. Furthermore, the expression of octamer-binding transcription factor 4, sex determining region Y-box 2 and Lin28B also significantly increased in gastric cancer cells treated with MSC-exosome. This present study indicates that MSC-exosome elicited this facilitation of migration and invasion in gastric cancer predominantly via the activation of the protein kinase B signaling pathway [59]. However, the bone marrow stromal cells (BMSCs) derived exosomes favor multiple myeloma (MM) cell migration, this study revealed that BMSC-exosomes selectively carry certain cytokines that produced by BMSCs such as chemotactic proteins MCP-1, MCP-2, MCP-3, 40 SDF-1, 41,42 and IGF-1, and transfer them to the recipient cells, to trigger MM cell migration, then induce migration of the 5T33MM cells [60].

On the other hand, MSC could also pack miRNA into exosomes and suppress tumor migration and invasion. Lee et al. reported that MSC could efficiently deliver synthetic miR-124 and miR-145 mimics to co-cultured glioma cells by exosomes via gap junction-dependent and independent processes. These delivered miR-124 and miR-145 mimics significantly decreased the migration of glioma cells because they decreased the luciferase activity of their respected reporter target genes SCP-1 and Sox2 [61]. Exosomes could also transfer extracellular miR-143 produced by MSC to osteosarcoma cells, which significantly reduced the migration of osteosarcoma cells [62]. In breast cancer, increased miR-23b and decreased MARCKS expression in exosomes secreted by bone marrow mesenchymal stem cells (BM-MSCs) contribute to cell cycle suppression and dormancy in breast cancer cells, which is one of the mechanisms result in inhibition of migration and invasion in breast cancer $[63,64]$.

To sum up, these results reveal that the MSC-exosomes can have different effects on same cancer, highlighting the necessity of tracking down 


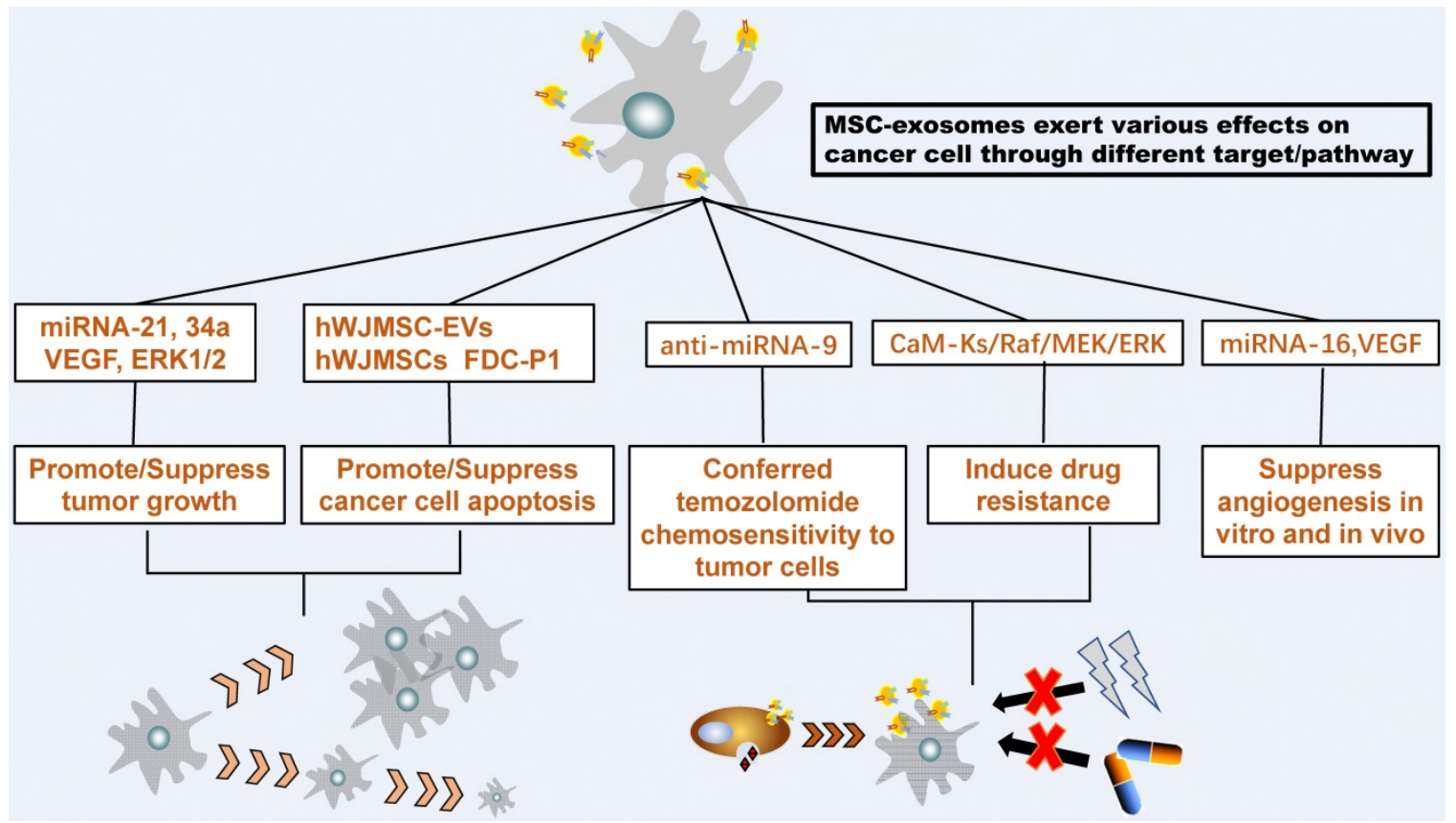

Figure 2. MSC-Exosome interact with tumor cells. MSC-exosomes could transfer proteins, messenger RNA, and microRNA to recipient tumor cells then exert various effects on the growth, metastasis, and drug response of different tumor cells

the mechanism of MSC-exosomes in various tumor cell types. An additional figure file shows this totally [see Figure 2].

\section{Transport Function and Novel Therapy of MSC-exosomes}

Exosomes are considered to be natural nanocarriers which have the absolute predominance in biocompatibility that can be used in clinical applications, such as drug delivery or transfer some specific mRNAs, regulatory miRNAs, lipids, and proteins $[65,66]$. Their role in cell-to-cell communication and because exogenous cargo can be loaded into them to deliver therapeutics to tumor sites $[67,68]$ provides therapeutic potential for cancer in future clinical medicine. For example, extracted exosomes from BM-MSCs transfected with miR-221 oligonucleotides can act as high-efficiency nanocarriers, which can provide sufficient miR-221 oligonucleotides to influence the tumor microenvironment and tumor aggressiveness effectively and promote oncogenic activity in gastric cancer [69]. In Glioma Stem-like Cells (GSCs), Glioma Associated-human Mesenchymal Stem Cells (GA-hMSCs) release exosomes contain highly expressed and highly enriched miR-1587, which is at least one exosomal miRNA that appears to mediate the increase in proliferation and clonogenicity of GSCs in vitro experiments. And the delivery of miR-1587 by GA-hMSCs-derived exosomes resulted in the down-regulation of the tumor-suppressor NCOR1 in the recipient GSCs, which increase the tumorigenicity of glioma stem-like cells and enhance the aggressiveness of glioblastoma [70]. On the other hand, due to their membrane composition and the adhesive proteins embedded within them, MSC-exosomes are perfect drug delivery vehicles, to deliver therapeutic agents such as therapeutic miRNA and anti-cancer agents [68]. A research shows that MSCs are able to package and deliver active drugs through their exosomes, this effect was tested on the human pancreatic cell line CFPAC-1, after priming with Paclitaxel (PTX) MSCs can acquire strong anti-tumor activity and release the drug through exosomes, verified the possibility of using MSC-exosomes as a carrier to develop drugs with a higher cell-target specificity [71].

Sometimes, after radiotherapy, chemotherapy, and surgery, the continued presence of small amounts of resistant cancer cells can lead to cancer recurrence. To change the number of untreated cancer cells, the residual tumor cells transfer resistance to sensitive cells through exosomes [72]. Exosomes from MSC derived from rat bone marrow can protect the rat pheochromocytoma PC12 cells against the excitotoxicity induced by glutamate [35]. It was discovered through this experiment that MSCs-exosomes reduced the expression of Bax and Bcl-2. At the same time, the researchers found that MSC-exosomes could reduce the sensitivity of BM2 cells to conventional chemotherapy drugs docetaxel [63]. And according to another experiment, MSC-exosomes could induce drug resistance in gastric cancer cells to 5-fluorouracil both in vivo and $e x$ 
vivo by activating CaM-Ks/Raf/MEK/ERK pathway [73]. In some researches, MSC-exosomes mediate drug efflux and transfer of drug resistance to recipient cells, through transferring of protein (MRP2, ATP7A, and ATP7B) and miRNAs (miR-100, miR - 222, miR 30a, miR-17). The miRNAs transfer to receptor cells can change the cell cycle and affect cell apoptosis, thus reducing the susceptibility of drugs. But in the meantime, a study report on a potential RNA therapy for the neuro-developmental miR-9 through the delivery of MSC-exosomes to make glioblastoma multiforme (GBM) sensitive to temozolomide (TMZ), which means delivery of synthetic anti-miR-9 by MSC-exosomes can reverse the chemoresistance of GBM cells [74]. Besides, exosomes derived from murine or human marrow MSC can reverse radiation injury to the murine bone marrow in vivo and in vitro. In addition, a study showed that vesicles stimulate proliferation and reverse radiation induced DNA damage and apoptosis in FDC-P1 cells [75].

\section{MSC-exosomes in Immunity}

Exosomes have been implicated in many aspects of immune regulation such as stimulation of $\mathrm{T}$ cell proliferation, B lymphocyte-mediated tumor suppression, induction of apoptosis in activated cytotoxic $\mathrm{T}$ cells, differentiation of monocytes into dendritic cells, and induction of myeloid-suppressive cells and $\mathrm{T}$ regulatory cells [76-79]. MSC-exosomes express galectin-1 and PD-L1 [80, 81], two molecules also expressed on MSC surface [82, 83]. Galectin-1, an endogenous leptin, has been shown to induce apoptosis of activated T cells [84] and to promote the generation of T regulatory cells [85]. PD-L1, a negative costimulatory molecule for PD-1, also promotes $\mathrm{T}$ regulatory cells proliferation and function [86-88]. Moreover, MSC-exosomes express TGF-b, a well-known inducer of $\mathrm{T}$ regulatory cells [83, 89, 90].

MSC-derived exosomes also inhibited proliferation of Concanavalin A-activated lymphocytes. This ability to exert suppressive and regulatory effects in an allogeneic or autologous manner would enhance the longevity of MSC exosome-derived drug delivery vehicle and bioavailability of its drug cargo [3].

\section{Conclusions}

In recent years, many researchers have found MSC-exosomes are playing a more and more important role in cancer cell-to-cell interaction in vivo and in vitro. As extracellular vesicles from MSC, exosomes are shown the most similarity of the beneficial and detrimental effects of the cells of origin, they can be carriers to transfer many kinds of molecules from MSC to recipient cells, and then activate a series of effects in cancer cell, which is the major way to suppress or support cancer development, and combine with the function that MSC-exosomes could influence the immunity such as leukomonocyte proliferation, an additional tabular file shows this contents in detail [see Table 1]. MSC-exosomes will be great biological tools for cancer therapy. However, despite increasing evidence for the therapeutic efficacy of MSC-derived exosomes, in the future study, it is hopeful to delve deeper into the potential of MSC-exosomes among cancer cells and provide effective treatments with the highest safety.

Table 1. The function of MSC-derived exosomes

\begin{tabular}{|c|c|c|c|}
\hline Source of Exosomes & Function & Target/Pathway & Reference \\
\hline $\begin{array}{l}\text { Human bone } \\
\text { marrow-derived } \\
\text { MSCs }\end{array}$ & $\begin{array}{l}\uparrow \text { Breast tumor } \\
\text { growth in vivo }\end{array}$ & miRNA-21 and 34a & [43] \\
\hline $\begin{array}{l}\text { Mouse bone } \\
\text { marrow-derived } \\
\text { MSCs }\end{array}$ & $\begin{array}{l}\downarrow \text { Suppress } \\
\text { angiogenesis in vitro } \\
\text { and in vivo }\end{array}$ & miRNA-16, VEGF & [44] \\
\hline $\begin{array}{l}\text { Human bone } \\
\text { marrow-derived } \\
\text { MSCs }\end{array}$ & $\begin{array}{l}\uparrow \text { Promote tumor } \\
\text { growth in vivo }\end{array}$ & VEGF, ERK1/2 & [39] \\
\hline $\begin{array}{l}\text { Mesenchymal } \\
\text { stromal } \\
\text { cell }\end{array}$ & $\begin{array}{l}\uparrow \text { Conferred } \\
\text { temozolomide } \\
\text { chemosensitivity to } \\
\text { tumor cells in vitro }\end{array}$ & anti-miRNA-9 & [74] \\
\hline $\begin{array}{l}\text { Mesenchymal } \\
\text { stromal } \\
\text { cell exosomes }\end{array}$ & $\begin{array}{l}\downarrow \text { Reduced } \\
\text { intracranial } \\
\text { tumor volume in } \\
\text { vivo }\end{array}$ & miRNA-146b & [47] \\
\hline $\begin{array}{l}\text { Multiple } \\
\text { myeloma-derived } \\
\text { MSCs }\end{array}$ & $\begin{array}{l}\uparrow \mathrm{MM} \text { cell growth in } \\
\text { vitro } \\
\uparrow T \text { Tumor growth in } \\
\text { vivo } \\
\uparrow \mathrm{BM} \text { homing }\end{array}$ & $\begin{array}{l}\text { MM BM-MSC-derived } \\
\text { exosomes }\end{array}$ & [41] \\
\hline $\begin{array}{l}\text { Human umbilical } \\
\text { cord Wharton's jelly } \\
\text { MSC }\end{array}$ & $\begin{array}{l}\downarrow \text { Significantly tumor } \\
\text { size } \\
\uparrow \text { Apoptosis }\end{array}$ & hWJMSC-EVs + hWJMSCs & [91] \\
\hline $\begin{array}{l}\text { Human adult liver } \\
\text { stem cell; }\end{array}$ & $\begin{array}{l}\downarrow \text { Significantly tumor } \\
\text { size } \\
\uparrow \text { Apoptosis }\end{array}$ & HLSC-derived exosomes & [92] \\
\hline BM MSC & $\begin{array}{l}\downarrow \text { Proliferation } \\
\downarrow \text { Tumor formation }\end{array}$ & $\begin{array}{l}\text { BM-MSC-derived } \\
\text { Exosome-treated cells }\end{array}$ & {$[63]$} \\
\hline $\begin{array}{l}\text { Glioma } \\
\text { Associated-human } \\
\text { MSC }\end{array}$ & $\begin{array}{l}\uparrow \text { increase the } \\
\text { tumorigenicity of } \\
\text { glioma stem-like } \\
\text { cells } \\
\uparrow \text { enhance the } \\
\text { aggressiveness of } \\
\text { glioblastoma }\end{array}$ & miR-1587 & {$[70]$} \\
\hline MSC & $\begin{array}{l}\downarrow \text { Induce drug } \\
\text { resistance } \\
\text { in gastric cancer cells }\end{array}$ & CaM-Ks/Raf/MEK/ERK & [73] \\
\hline $\begin{array}{l}\text { murine or human } \\
\text { marrow MSC }\end{array}$ & $\begin{array}{l}\downarrow \text { reverse radiation } \\
\text { injury to murine } \\
\text { bone marrow in vivo } \\
\text { and in vitro } \\
\downarrow \text { Apoptosis }\end{array}$ & FDC-P1 & {$[75]$} \\
\hline
\end{tabular}

\section{Abbreviations}

MSCs: Mesenchymal stem (or stromal) cells

TGF- $\beta$ : transforming growth factor- $\beta$

PD-1: programmed cell death protein 1

PD-L1: Programmed death-ligand 1

GBM: glioblastoma multiforme 
TMZ: temozolomide

PTX: Paclitaxel

Glioma

Associated-human

Mesenchymal Stem Cells

GSCs: Glioma Stem-like Cells

BM-MSCs: bone marrow mesenchymal stem cells

BMSCs: bone marrow stromal cells

MM: multiple myeloma

GC: gastric cancer

VEGF: Vascular endothelial growth factor

MMPs: matrix metalloproteases

HIF-1 $\alpha$ : hypoxia-inducible factor

ROS: reactive oxygen species

IL-6: interleukin-6

\section{Acknowledgements}

\section{Funding}

This work was supported by the National Natural Science Foundation (81202115), Doctoral Innovation Fund of Shanghai Jiaotong University School of Medicine (No. BXJ201732).

\section{Authors' contributions}

JZ conceptualized the study, performed the literature search, and drafted most of the manuscript. XT and YT made the table. YT and QL wrote the section on tumor angiogenesis and MSC-exosomes in Immunity. GW revised the article and directed the review to be more focused. JM gave final approval for the article to be published. All authors read and approved the manuscript.

\section{Competing Interests}

The authors have declared that no competing interest exists.

\section{References}

1. Barry F, Murphy J. Mesenchymal stem cells: clinical applications and biological characterization. Int J Biochem Cell Biol. 2004; 36: 568-84.

2. Giebel B, Kordelas L, Börger V. Clinical potential of mesenchymal stem/stromal cell-derived extracellular vesicles. Stem Cell Investig. 2017; 4: 84

3. Yeo RWY, Lai RC, Zhang B, Tan SS, Yin Y, Teh BJ, et al. Mesenchymal stem cell: An efficient mass producer of exosomes for drug delivery. Advanced Drug Delivery Reviews. 2013; 65: 336-41.

4. Banas A, Teratani T, Yamamoto Y, Tokuhara M, Takeshita F, Quinn G, et al. Adipose tissue-derived mesenchymal stem cells as a source of human hepatocytes. Hepatology. 2007; 46: 219-28.

5. Lai R, Arslan F, Tan S, Tan B, Choo A, Lee M, et al. Derivation and characterization of human fetal MSCs: an alternative cell source for large-scale production of cardioprotective microparticles. J Mol Cell Cardiol. 2010; 48: 1215-24

6. Fukuchi Y, Nakajima H, Sugiyama D, Hirose I, Kitamura T, Tsuji K. Human placenta-derived cells have mesenchymal stem/progenitor cell potential. Stem Cells. 2004; 22: 649-58.

7. Miao Z, Jin J, Chen L, Zhu J, Huang W, Zhao J, et al. Isolation of mesenchymal stem cells from human placenta: comparison with human bone marrow mesenchymal stem cells. Cell Biol Int. 2006; 30: 681-7.

8. in 't Anker P, Noort W, Scherjon S, Kleijburg-van der Keur C, Kruisselbrink A van Bezooijen R, et al. Mesenchymal stem cells in human second-trimester bone marrow, liver, lung, and spleen exhibit a similar immunophenotype but a heterogeneous multilineage differentiation potential. Haematologica. 2003; 88: 845-52.
9. Arai F, Ohneda O, Miyamoto T, Zhang X, Suda T. Mesenchymal stem cells in perichondrium express activated leukocyte cell adhesion molecule and participate in bone marrow formation. J Exp Med. 2002; 195: 1549-63.

10. Young $\mathrm{H}$, Steele $\mathrm{T}$, Bray $\mathrm{R}$, Hudson J, Floyd J, Hawkins $\mathrm{K}$, et al. Human reserve pluripotent mesenchymal stem cells are present in the connective tissues of skeletal muscle and dermis derived from fetal, adult, and geriatric donors. Anat Rec. 2001; 264: 51-62.

11. Roubelakis M, Pappa K, Bitsika V, Zagoura D, Vlahou A, Papadaki H, et al. Molecular and proteomic characterization of human mesenchymal stem cells derived from amniotic fluid: comparison to bone marrow mesenchymal stem cells. Stem Cells Dev. 2007; 16: 931-52.

12. Le Blanc K, Tammik L, Sundberg B, Haynesworth S, Ringdén O. Mesenchymal stem cells inhibit and stimulate mixed lymphocyte cultures and mitogenic responses independently of the major histocompatibility complex. Scand J Immunol. 2003; 57: 11-20.

13. Bartholomew A, Sturgeon C, Siatskas M, Ferrer K, McIntosh K, Patil S, et al. Mesenchymal stem cells suppress lymphocyte proliferation in vitro and prolong skin graft survival in vivo. Exp Hematol. 2002; 30: 42-8.

14. Beach A, Zhang H, Ratajczak M, Kakar S. Exosomes: an overview of biogenesis, composition and role in ovarian cancer. J Ovarian Res. 2014; 7: 14.

15. Taylor D, Gercel-Taylor C. MicroRNA signatures of tumor-derived exosomes as diagnostic biomarkers of ovarian cancer. Gynecol Oncol. 2008; 110: 13-21.

16. Simpson R, Lim J, Moritz R, Mathivanan S. Exosomes: proteomic insights and diagnostic potential. Expert Rev Proteomics. 2009; 6: 267-83.

17. Gallo A, Tandon M, Alevizos I, Illei G. The majority of microRNAs detectable in serum and saliva is concentrated in exosomes. PLoS ONE. 2012; 7: e30679.

18. Hemler M. Tetraspanin proteins mediate cellular penetration, invasion, and fusion events and define a novel type of membrane microdomain. Annu Rev Cell Dev Biol. 2003; 19: 397-422.

19. Mathivanan S, Fahner C, Reid G, Simpson R. ExoCarta 2012: database of exosomal proteins, RNA and lipids. Nucleic Acids Res. 2012; 40: D1241-4.

20. Mathivanan S, Simpson R. ExoCarta: A compendium of exosomal proteins and RNA. Proteomics. 2009; 9: 4997-5000.

21. $\mathrm{Li} X$, Wang $X$. The emerging roles and therapeutic potential of exosomes in epithelial ovarian cancer. Mol Cancer. 2017; 16: 92

22. Ogorevc E, Kralj-Iglic V, Veranic P. The role of extracellular vesicles in phenotypic cancer transformation. Radiol Oncol. 2013; 47: 197-205.

23. Xiang X, Poliakov A, Liu C, Liu Y, Deng Z, Wang J, et al. Induction of myeloid-derived suppressor cells by tumor exosomes. Int J Cancer. 2009; 124: 2621-33.

24. Yeo RW, Lai RC, Zhang B, Tan SS, Yin Y, Teh BJ, et al. Mesenchymal stem cell: an efficient mass producer of exosomes for drug delivery. Adv Drug Deliv Rev. 2013; 65: 336-41

25. Lai R, Arslan F, Lee M, Sze N, Choo A, Chen T, et al. Exosome secreted by MSC reduces myocardial ischemia/reperfusion injury. Stem Cell Res. 2010; 4: 214-22.

26. Nakamura Y, Miyaki S, Ishitobi H, Matsuyama S, Nakasa T, Kamei N, et al. Mesenchymal-stem-cell-derived exosomes accelerate skeletal muscle regeneration. FEBS Lett. 2015; 589: 1257-65.

27. Bai L, Shao H, Wang H, Zhang Z, Su C, Dong L, et al. Effects of Mesenchymal Stem Cell-Derived Exosomes on Experimental Autoimmune Uveitis. Sci Rep. 2017; 7: 4323.

28. Sun L, Li D, Song K, Wei J, Yao S, Li Z, et al. Exosomes derived from human umbilical cord mesenchymal stem cells protect against cisplatin-induced ovarian granulosa cell stress and apoptosis in vitro. Sci Rep. 2017; 7: 2552.

29. Pachler K, Ketterl N, Desgeorges A, Dunai ZA, Laner-Plamberger S, Streif D, et al. An In Vitro Potency Assay for Monitoring the Immunomodulatory Potential of Stromal Cell-Derived Extracellular Vesicles. Int J Mol Sci. 2017; 18.

30. Yu B, Zhang X, Li X. Exosomes derived from mesenchymal stem cells. Int J Mol Sci. 2014; 15: 4142-57

31. Gong M, Yu B, Wang J, Wang Y, Liu M, Paul C, et al. Mesenchymal stem cells release exosomes that transfer miRNAs to endothelial cells and promote angiogenesis. Oncotarget. 2017; 8: 45200-12.

32. Chan J, Krichevsky A, Kosik K. MicroRNA-21 is an antiapoptotic factor in human glioblastoma cells. Cancer Res. 2005; 65: 6029-33.

33. Lai R, Tan S, Teh B, Sze S, Arslan F, de Kleijn D, et al. Proteolytic Potential of the MSC Exosome Proteome: Implications for an Exosome-Mediated Delivery of Therapeutic Proteasome. Int J Proteomics. 2012; 2012: 971907.

34. Suchorska W, Lach M. The role of exosomes in tumor progression and metastasis (Review). Oncol Rep. 2016; 35: 1237-44.

35. Zhang X, Tu H, Yang Y, Fang L, Wu Q, Li J. Mesenchymal Stem Cell-Derived Extracellular Vesicles: Roles in Tumor Growth, Progression, and Drug Resistance. Stem Cells Int. 2017; 2017: 1758139.

36. Ratajczak J, Miekus K, Kucia M, Zhang J, Reca R, Dvorak P, et al. Embryonic stem cell-derived microvesicles reprogram hematopoietic progenitors: evidence for horizontal transfer of mRNA and protein delivery. Leukemia. 2006; 20: 847-56.

37. Grange C, Tapparo M, Collino F, Vitillo L, Damasco C, Deregibus M, et al. Microvesicles released from human renal cancer stem cells stimulate angiogenesis and formation of lung premetastatic niche. Cancer Res. 2011; 71: 5346-56

38. Camussi G, Deregibus M, Bruno S, Cantaluppi V, Biancone L. Exosomes/microvesicles as a mechanism of cell-to-cell communication. Kidney Int. 2010; 78: 838-48 
39. Zhu W, Huang L, Li Y, Zhang X, Gu J, Yan Y, et al. Exosomes derived from human bone marrow mesenchymal stem cells promote tumor growth in vivo. Cancer Lett. 2012; 315: 28-37.

40. Qi J, Zhou Y, Jiao Z, Wang X, Zhao Y, Li Y, et al. Exosomes Derived from Human Bone Marrow Mesenchymal Stem Cells Promote Tumor Growth Through Hedgehog Signaling Pathway. Cell Physiol Biochem. 2017; 42: 2242-54

41. Roccaro A, Sacco A, Maiso P, Azab A, Tai Y, Reagan M, et al. BM mesenchymal stromal cell-derived exosomes facilitate multiple myeloma progression. J Clin Invest. 2013; 123: 1542-55.

42. Yang Y, Bucan V, Baehre H, von der Ohe J, Otte A, Hass R. Acquisition of new tumor cell properties by MSC-derived exosomes. Int J Oncol. 2015; 47: 244-52.

43. Vallabhaneni K, Penfornis P, Dhule S, Guillonneau F, Adams K, Mo Y, et al. Extracellular vesicles from bone marrow mesenchymal stem/stromal cells transport tumor regulatory microRNA, proteins, and metabolites. Oncotarget. 2015; 6: 4953-67.

44. Lee J, Park S, Jung B, Jeon Y, Lee Y, Kim M, et al. Exosomes derived from mesenchymal stem cells suppress angiogenesis by down-regulating VEGF expression in breast cancer cells. PLoS ONE. 2013; 8: e84256.

45. Pakravan K, Babashah S, Sadeghizadeh M, Mowla S, Mossahebi-Mohammadi M, Ataei F, et al. MicroRNA-100 shuttled by mesenchymal stem cell-derived exosomes suppresses in vitro angiogenesis through modulating the mTOR/HIF-1a/VEGF signaling axis in breast cancer cells. Cell Oncol (Dordr). 2017.

46. Katakowski M, Zheng X, Jiang F, Rogers T, Szalad A, Chopp M. MiR-146b-5p suppresses EGFR expression and reduces in vitro migration and invasion of glioma. Cancer Invest. 2010; 28: 1024-30.

47. Katakowski M, Buller B, Zheng X, Lu Y, Rogers T, Osobamiro O, et al. Exosomes from marrow stromal cells expressing miR-146b inhibit glioma growth. Cancer Lett. 2013; 335: 201-4.

48. Del Fattore A, Luciano R, Saracino R, Battafarano G, Rizzo C, Pascucci L, et al. Differential effects of extracellular vesicles secreted by mesenchymal stem cells from different sources on glioblastoma cells. Expert Opin Biol Ther. 2015; 15: 495-504.

49. Sharma A. Role of stem cell derived exosomes in tumor biology. Int J Cancer. 2018; 142: 1086-92

50. Skog J, Würdinger T, van Rijn S, Meijer D, Gainche L, Sena-Esteves M, et al. Glioblastoma microvesicles transport RNA and proteins that promote tumour growth and provide diagnostic biomarkers. Nat Cell Biol. 2008; 10: 1470-6.

51. Thompson C, Purushothaman A, Ramani V, Vlodavsky I, Sanderson R. Heparanase regulates secretion, composition, and function of tumor cell-derived exosomes. J Biol Chem. 2013; 288: 10093-9.

52. Koch S, Claesson-Welsh L. Signal transduction by vascular endothelial growth factor receptors. Cold Spring Harb Perspect Med. 2012; 2: a006502.

53. Kim Y, Byzova T. Oxidative stress in angiogenesis and vascular disease. Blood. 2014; 123: 625-31.

54. Szatrowski T, Nathan C. Production of large amounts of hydrogen peroxide by human tumor cells. Cancer Res. 1991; 51: 794-8.

55. Lopatina T, Gai C, Deregibus M, Kholia S, Camussi G. Cross Talk between Cancer and Mesenchymal Stem Cells through Extracellular Vesicles Carrying Nucleic Acids. Front Oncol. 2016; 6: 125

56. Lin R, Wang S, Zhao R. Exosomes from human adipose-derived mesenchymal stem cells promote migration through Wnt signaling pathway in a breast cancer cell model. Mol Cell Biochem. 2013; 383: 13-20.

57. Wang M, Zhao $\mathrm{C}$, Shi $\mathrm{H}$, Zhang B, Zhang L, Zhang $\mathrm{X}$, et al. Deregulated microRNAs in gastric cancer tissue-derived mesenchymal stem cells: novel biomarkers and a mechanism for gastric cancer. Br J Cancer. 2014; 110: 1199-210.

58. Liu K, Li G, Fan C, Diao Y, Wu B, Li J. Increased Expression of MicroRNA-221 in gastric cancer and its clinical significance. J Int Med Res. 2012; 40: 467-74.

59. Gu H, Ji R, Zhang X, Wang M, Zhu W, Qian H, et al. Exosomes derived from human mesenchymal stem cells promote gastric cancer cell growth and migration via the activation of the Akt pathway. Mol Med Rep. 2016; 14: 3452-8.

60. Wang J, Hendrix A, Hernot S, Lemaire M, De Bruyne E, Van Valckenborgh E, et al. Bone marrow stromal cell-derived exosomes as communicators in drug resistance in multiple myeloma cells. Blood. 2014; 124: 555-66.

61. Lee H, Finniss S, Cazacu S, Bucris E, Ziv-Av A, Xiang C, et al. Mesenchymal stem cells deliver synthetic microRNA mimics to glioma cells and glioma stem cells and inhibit their cell migration and self-renewal. Oncotarget. 2013; 4: $346-61$.

62. Shimbo K, Miyaki S, Ishitobi $\mathrm{H}$, Kato $\mathrm{Y}$, Kubo $\mathrm{T}$, Shimose $\mathrm{S}$, et al. Exosome-formed synthetic microRNA-143 is transferred to osteosarcoma cells and inhibits their migration. Biochem Biophys Res Commun. 2014; 445: 381-7.

63. Ono M, Kosaka N, Tominaga N, Yoshioka Y, Takeshita F, Takahashi R, et al. Exosomes from bone marrow mesenchymal stem cells contain a microRNA that promotes dormancy in metastatic breast cancer cells. Sci Signal. 2014; 7: ra63.

64. Bliss SA, Sinha G, Sandiford OA, Williams LM, Engelberth DJ, Guiro K, et al. Mesenchymal Stem Cell-Derived Exosomes Stimulate Cycling Quiescence and Early Breast Cancer Dormancy in Bone Marrow. Cancer Res. 2016; 76: 5832-44.

65. Syn N, Wang L, Chow E, Lim C, Goh B. Exosomes in Cancer Nanomedicine and Immunotherapy: Prospects and Challenges. Trends Biotechnol. 2017; 35: $665-76$
66. Luan X, Sansanaphongpricha K, Myers I, Chen H, Yuan H, Sun D. Engineering exosomes as refined biological nanoplatforms for drug delivery. Acta Pharmacol Sin. 2017; 38: 754-63.

67. Batrakova E, Kim M. Using exosomes, naturally-equipped nanocarriers, for drug delivery. J Control Release. 2015; 219: 396-405.

68. Moore C, Kosgodage U, Lange S, Inal J. The emerging role of exosome and microvesicle- (EMV-) based cancer therapeutics and immunotherapy. Int J Cancer. 2017; 141: 428-36.

69. Ma M, Chen S, Liu Z, Xie H, Deng H, Shang S, et al. miRNA-221 of exosomes originating from bone marrow mesenchymal stem cells promotes oncogenic activity in gastric cancer. Onco Targets Ther. 2017; 10: 4161-71.

70. Figueroa J, Phillips L, Shahar T, Hossain A, Gumin J, Kim H, et al. Exosomes from Glioma-Associated Mesenchymal Stem Cells Increase the Tumorigenicity of Glioma Stem-like Cells via Transfer of miR-1587. Cancer Res. 2017.

71. Pascucci L, Coccè V, Bonomi A, Ami D, Ceccarelli P, Ciusani E, et al. Paclitaxel is incorporated by mesenchymal stromal cells and released in exosomes that inhibit in vitro tumor growth: a new approach for drug delivery. J Control Release. 2014; 192: 262-70

72. Chen W, Liu X, Lv M, Chen L, Zhao J, Zhong S, et al. Exosomes from drug-resistant breast cancer cells transmit chemoresistance by a horizontal transfer of microRNAs. PLoS ONE. 2014; 9: e95240.

73. Ji R, Zhang B, Zhang X, Xue J, Yuan X, Yan Y, et al. Exosomes derived from human mesenchymal stem cells confer drug resistance in gastric cancer. Cell Cycle. 2015; 14: 2473-83.

74. Munoz J, Bliss S, Greco S, Ramkissoon S, Ligon K, Rameshwar P. Delivery of Functional Anti-miR-9 by Mesenchymal Stem Cell-derived Exosomes to Glioblastoma Multiforme Cells Conferred Chemosensitivity. Mol Ther Nucleic Acids. 2013; 2: e126.

75. Wen S, Dooner M, Cheng Y, Papa E, Del Tatto M, Pereira M, et al. Mesenchymal stromal cell-derived extracellular vesicles rescue radiation damage to murine marrow hematopoietic cells. Leukemia. 2016; 30: 2221-31.

76. Taylor D, Gercel-Taylor C. Exosomes/microvesicles: mediators of cancer-associated immunosuppressive microenvironments. Semin Immunopathol. 2011; 33: 441-54

77. Bobrie A, Colombo M, Raposo G, Théry C. Exosome secretion: molecular mechanisms and roles in immune responses. Traffic. 2011; 12: 1659-68.

78. György B, Szabó T, Pásztói M, Pál Z, Misják P, Aradi B, et al. Membrane vesicles, current state-of-the-art: emerging role of extracellular vesicles. Cell Mol Life Sci. 2011; 68: 2667-88.

79. Chaput N, Théry C. Exosomes: immune properties and potential clinical implementations. Semin Immunopathol. 2011; 33: 419-40.

80. Garín M, Chu C, Golshayan D, Cernuda-Morollón E, Wait R, Lechler R. Galectin-1: a key effector of regulation mediated by CD4+CD25+ T cells. Blood. 2007; 109: 2058-65.

81. Kilpinen L, Impola U, Sankkila L, Ritamo I, Aatonen M, Kilpinen S, et al. Extracellular membrane vesicles from umbilical cord blood-derived MSC protect against ischemic acute kidney injury, a feature that is lost after inflammatory conditioning. J Extracell Vesicles. 2013; 2.

82. Kadri T, Lataillade J, Doucet C, Marie A, Ernou I, Bourin P, et al. Proteomic study of Galectin-1 expression in human mesenchymal stem cells. Stem Cells Dev. 2005; 14: 204-12.

83. Pedemonte E, Benvenuto F, Casazza S, Mancardi G, Oksenberg J, Uccelli A, et al. The molecular signature of therapeutic mesenchymal stem cells exposes the architecture of the hematopoietic stem cell niche synapse. BMC Genomics. 2007; 8: 65.

84. Rabinovich G, Alonso C, Sotomayor C, Durand S, Bocco J, Riera C. Molecular mechanisms implicated in galectin-1-induced apoptosis: activation of the AP-1 transcription factor and downregulation of Bcl-2. Cell Death Differ. 2000; 7: 747-53.

85. Blois S, Ilarregui J, Tometten M, Garcia M, Orsal A, Cordo-Russo R, et al. A pivotal role for galectin-1 in fetomaternal tolerance. Nat Med. 2007; 13: $1450-7$.

86. Francisco L, Salinas V, Brown K, Vanguri V, Freeman G, Kuchroo V, et al. PD-L1 regulates the development, maintenance, and function of induced regulatory T cells. J Exp Med. 2009; 206: 3015-29.

87. Freeman G, Long A, Iwai Y, Bourque K, Chernova T, Nishimura H, et al. Engagement of the PD-1 immunoinhibitory receptor by a novel B7 family member leads to negative regulation of lymphocyte activation. J Exp Med. 2000; 192: 1027-34

88. Guleria I, Khosroshahi A, Ansari M, Habicht A, Azuma M, Yagita H, et al. A critical role for the programmed death ligand 1 in fetomaternal tolerance. J Exp Med. 2005; 202: 231-7.

89. Chen W, Jin W, Hardegen N, Lei K, Li L, Marinos N, et al. Conversion of peripheral CD4+CD25- naive $\mathrm{T}$ cells to $\mathrm{CD} 4+\mathrm{CD} 25+$ regulatory $\mathrm{T}$ cells by TGF-beta induction of transcription factor Foxp3. J Exp Med. 2003; 198: $1875-86$

90. Fantini M, Becker C, Monteleone G, Pallone F, Galle P, Neurath M. Cutting edge: TGF-beta induces a regulatory phenotype in CD4+CD25- T cells through Foxp3 induction and down-regulation of Smad7. J Immunol. 2004; 172: 5149-53.

91. Wu S, Ju G, Du T, Zhu Y, Liu G. Microvesicles derived from human umbilical cord Wharton's jelly mesenchymal stem cells attenuate bladder tumor cell growth in vitro and in vivo. PLoS ONE. 2013; 8: e61366. 
92. Fonsato V, Collino F, Herrera M, Cavallari C, Deregibus M, Cisterna B, et al. Human liver stem cell-derived microvesicles inhibit hepatoma growth in SCID mice by delivering antitumor microRNAs. Stem Cells. 2012; 30: 1985-98. 